\title{
Improved wound management by regulated negative pressure-assisted wound therapy and regulated, oxygen-enriched negative pressure-assisted wound therapy through basic science research and clinical assessment
}

\section{Moris Topaz}

Plastic Surgery Unit, Hillel Yaffe Medical Center, Hadera, Department of Chemistry, Bar Ilan University, Ramat Gan, Israel

Address for correspondence: Dr. Moris Topaz, 16 Hatidhar St., Ra'anana, Israel. E-mail: topazmd@netvision.net.il

\section{ABSTRACT}

Regulated negative pressure-assisted wound therapy (RNPT) should be regarded as a state-of-theart technology in wound treatment and the most important physical, nonpharmaceutical, platform technology developed and applied for wound healing in the last two decades. RNPT systems maintain the treated wound's environment as a semi-closed, semi-isolated system applying external physical stimulations to the wound, leading to biological and biochemical effects, with the potential to substantially influence wound-host interactions, and when properly applied may enhance wound healing. RNPT is a simple, safe, and affordable tool that can be utilized in a wide range of acute and chronic conditions, with reduced need for complicated surgical procedures, and antibiotic treatment. This technology has been shown to be effective and safe, saving limbs and lives on a global scale. Regulated, oxygen-enriched negative pressure-assisted wound therapy (RO-NPT) is an innovative technology, whereby supplemental oxygen is concurrently administered with RNPT for their synergistic effect on treatment and prophylaxis of anaerobic wound infection and promotion of wound healing. Understanding the basic science, modes of operation and the associated risks of these technologies through their fundamental clinical mechanisms is the main objective of this review.

\section{KEY WORDS}

Regulated negative pressure-assisted wound therapy; regulated; oxygen-enriched negative pressure-assisted wound therapy; vacuum; wound healing

\section{INTRODUCTION}

\begin{tabular}{|l|l|}
\multicolumn{3}{|c|}{ Access this article online } \\
\hline Quick Response Code: & Website: \\
\hline & www.ijps.org \\
\cline { 2 - 2 } & Dol: \\
\hline
\end{tabular}

ound infection was once a major cause
of disability and mortality. However, the
introduction of antiseptic techniques and
antibiotics has dramatically changed the course of
wound healing. Today, however, wound healing is of even
greater global medical and financial significance because
of advancements in technologies, high-speed traffic
Indian Journal of Plastic Surgery May-August 2012 Vol 45 Issue 2 
accidents, modern weaponry's destructive capacity, the extension of life-expectancy, the increase in chronic diseases and the surging worldwide prevalence of diabetes, as well as the problem of antibiotic resistance. Any substantial progress in wound healing is expected to lead to a considerable improvement in health on a global scale.

RNPT utilizes subatmospheric pressure (vacuum, negative pressure) to optimize the wound's microenvironment to promote the healing process. This unique, physical, nonpharmaceutical technology applies regulated subatmospheric pressure to a wound, inflicting a variety of phenomena with the desired end-point of improved healing of simple and hard-to-cure, acute and chronic wounds. Through multiple publications on its advantages in various clinical applications during the last decade, RNPT has been established as an important technology, yet the full scope of the basic science and its associated mechanism of action, modes of application, and safe use need to be explored further. ${ }^{[1]}$

At the core of RNPT is the application of negative pressure, generated by a pump and controlled and regulated by a computerized program through predetermined mode settings of the applied negative pressure to the wound. The predetermined modulations of the basic functions of the vacuum source: the pressure level that is being applied to the wound, the mode of application (intermittent, continuous, or cyclic-continuous) with appropriate time intervals, as well as safety measures (sensors and alarms for exudate flow and vacuum levels), are of major importance in determining RNPT's advantages. Negative pressure is directed through tubing to a flexible open cell foam mold with pores in the range of $100-600 \mu \mathrm{m}$, which covers or is embedded in the wound. The multiporous, compressible mold is sealed with an air-tight drape, isolating the wound from the atmosphere above, and applying near-uniform subatmospheric pressure to the entire wound surface area [Figure 1].

Wound infection is a major impediment to healing. Allchronic wounds are colonized by bacteria, often without systemic clinical manifestation and significance when a low level of bacteria is involved; yet infection may play a significant role in wound healing when high-level bacterial counts subsist with deep invasion. Host-microbial interactions determine the progression of colonization to tissue invasion, infection, local damage, septicemia, and sepsis. Diverse microbial flora invade the wound bed of chronic nonhealing wounds, initially Gram-positive skin contaminants and gradually changing to various pathogenic species including aerobic Gram-negative rods as well as anaerobic bacteria, some in complex communities of aggregated bacteria embedded in an extracellular polymeric matrix of polysaccharides, forming a barrier defined as biofilm.

Indications for RNPT have widened during the last decade and now include a wide range of: chronic wounds (wounds in diabetic and PVD-affected limbs, decubitus ulcers), dehisced and infected surgical wounds, traumatic wounds, deep and partial-thickness small-to-medium size burns or wide area burns treated in increments, and treatment of skin grafts and flaps.

It is essential to improve this technology, widen its scope of applications, and lessen its intrinsic risks by better understanding the scientific basis ofits mechanisms through systematic basic science research, observant clinical application, analysis of patient-risk factors, and careful determination of its limitations and contraindications for certain clinical conditions and wound types.

\section{The physical basis of RNPT}

RNPT affects tissue through multiple, at times conflicting, physical effects of suction forces, topical pressure, shearing forces, and modification of the wound's atmospheric composition. These combined mechanisms trigger complex environmental, physical, chemical, and biological conditions that affect and determine wound healing. It is imperative to fully understand these physical mechanisms in an integrated manner due to their implications on various clinical conditions.

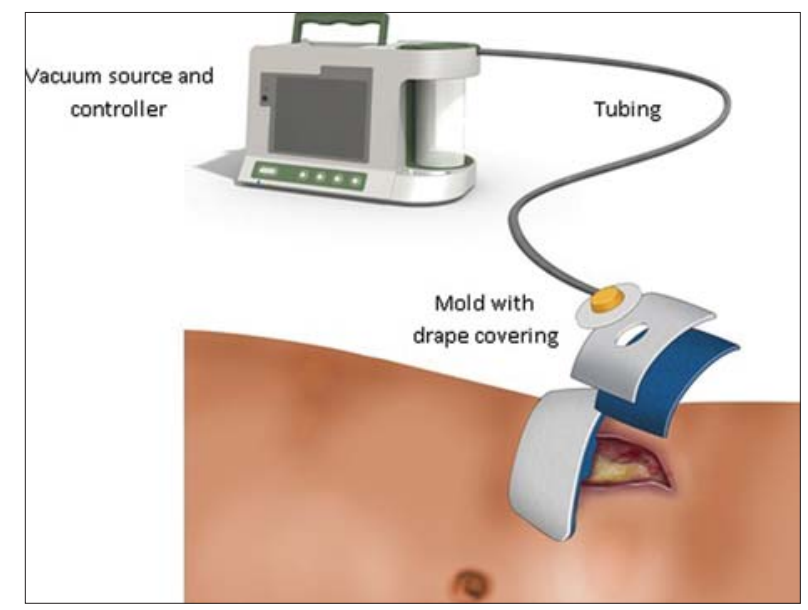

Figure 1: The basic concept of the RNPT system is illustrated: the application of preset computer-regulated negative pressure, generated by a pump, led by tubing to contact with the wound surface by a dressing or a porous compressible mold sponge covered, and is air-tight sealed by a drape 


\section{Suction forces}

Suction forces created by RNPT lead to a pressure gradient between the top-sealed open cell mold atmosphere and its attaching wound surface. These suction forces, applied into the wound cavity, have been reported to reverse lymphatic flow, reduce bacterial count, evacuate wound fluids, decompress tissue oedema, and induce granulation tissue formation. ${ }^{[2]}$

\section{Reversal of lymphatic flow}

Bacterial clearance was claimed to result from a decrease in wound surface area in vacuum-treated wounds. Case reports also showed a significant decrease in nonfermented Gram-negative rods (e.g., Pseudomonas) in biopsy specimens harvested from vacuum-treated wounds. ${ }^{[3]}$ In a study by Wagner et al., examining growth factor levels in acute and inflamed wounds, case reports showed positive smear cultures in inflamed wounds before vacuum treatment but negative smear cultures during vacuum treatment. ${ }^{[4]}$

The effect of negative pressure on tissue's lymphatic flow was demonstrated in our laboratory in a rabbit model by subcutaneous injection of methylene blue at various distances from the wound edges and evaluating the lymphatic flow distribution pattern [Figure 2]. A distortion from the regular elliptical pattern of lymphatic flow away from the wound, towards regional lymph nodes was demonstrated by the application of RNPT to the wound. Dye injected in close proximity to the wound's margins demonstrated a reverse flow pattern with a shift towards the wound, indicating a reversal of lymphatic flow from the surroundings of the wound towards the low pressure source.

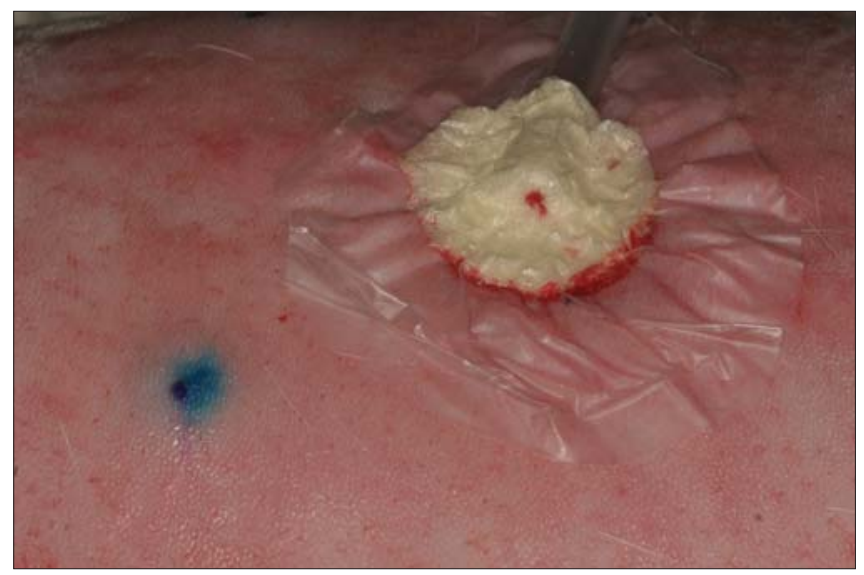

Figure 2: Reversal of lymphatic flow pattern in experimental RNPT treatment in a rabbit model. Wound is covered with an RNPT system, reversing the flow of the injected dye by suction forces to spread towards the vacuum source instead of drifting towards regional lymph nodes
This basic mechanism of reversal of lymphatic flow induced by suction forces during RNPT should be considered of significant clinical value, better elucidating the mechanism of bacterial clearance from the wound, the limitation of bacterial spread, and the rationale of the need for early initiation of RNPT to avoid dissemination of infection, and explaining the clinical observation of the reduced need for antibiotic treatment associated with RNPT.

\section{Induction of granulation tissue formation}

The most prominent reported effect of RNPT is the rapid growth of granulation tissue in the wound. RNPT was reported to induce and accelerate granulation tissue formation in porcine and rabbit wound models..$^{[5,6]}$

Studies examining the rate of granulation tissue formation in swine wounds showed that the $125 \mathrm{mmHg}$ pressuretreated wound filled with granulation tissue significantly faster than $25 \mathrm{mmHg}$ and $500-\mathrm{mmHg}$ treated wounds. ${ }^{17]}$ RNPT may induce granulation tissue to cover exposed bones and tendons, enabling coverage with skin grafts. ${ }^{[2]}$

\section{Evacuation of wound fluids and decompression of tissue oedema}

Crush injuries, complex fractures, acute vascular ischaemia, and deep burns may be associated with self-perpetuating inflammatory response, soft tissue oedema, and increased interstitial pressure. The integrity of the capillary basement membrane of blood vessels is damaged during wounding, leading to increased permeability of capillaries and oedema, followed by decreased peripheral perfusion and ischaemia as seen in compartment syndrome. Chen et al. reported restoration of the integrity of the basement membrane and narrowing of the endothelial spaces following RNPT. ${ }^{[8]}$ RNPT may have a role in the treatment of small- and medium-sized deep burns or partial treatment of big burns, by decongestion of oedema in the zone of stasis, improving the chances for recovery of tissue. RNPT can be applied in conjunction with surgical decompression of compartment syndrome and be applied directly on the fasciotomy wounds to further decompress the limb by sucking out fluid,and assisting in decompression of the internal tension (clinical cases, case 2). Initiation of RNPT at an early stage of injury is important to reduce infection.

\section{Topical pressure}

Application of subatmospheric pressure through a mold to the wound was shown, by laser Doppler measurements, to result in an immediate increase in tissue perfusion and 
was claimed to be an important factor accounting for the favorable effects of RNPT. ${ }^{[5,7]}$

This hypothesis was questioned and challenged by our in vitro and in vivo experimental findings of the topical pressure inflicted on tissue beneath circumferential and noncircumferential RNPT dressings on both an artificial and a live lower limb models, simulating clinical application of RNPT. We detected a near-linear rise of topical pressure beneath the dressings by increasing negative pressure of RNPT [Figure 3]. Pressure transducers were located at proximal and distal lower extremities of healthy volunteers and artificial leg model. Pressure gauges were placed between the skin and the mold to monitor the internal topical pressure induced by the increasing negative pressure. A near-linear rise of internal pressure beneath the mold was observed.

These findings contradict the reported increase of blood flow in wounds treated by RNPT and suggest the relative

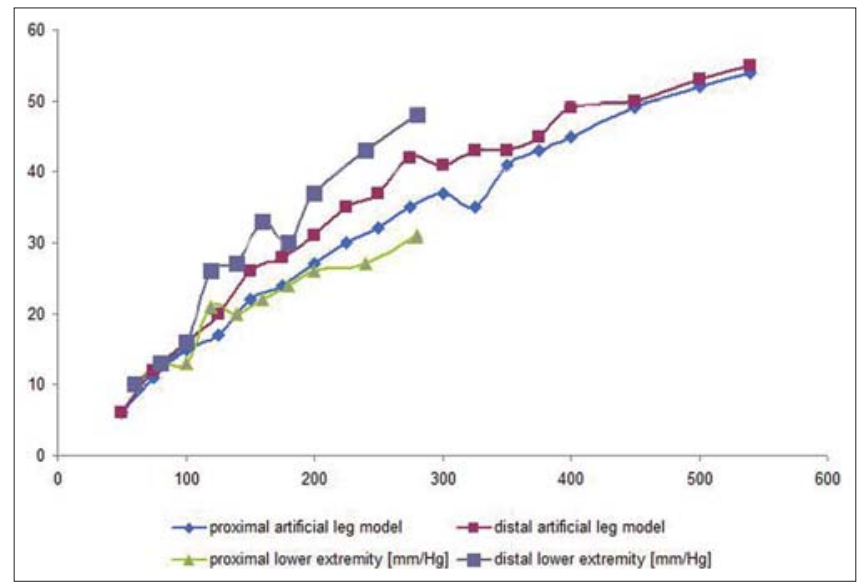

Figure 3: Experimental graphs demonstrating the relations between the negative pressure applied to the wound and the topical pressure inflicted by circumferential dressing of RNPT underneath the dressings in simulated lower limb and artificial lower limb wound models reduced perfusion that may lead to local ischaemia inflicted by the topical pressure underneath the RNPT dressings.

The actual effects of the pressure inflicted under RNPT system dressings may be further determined by the tissue plasticity, the underlying skeletal structure, presence of foreign bodies and their pliability, the physical characteristics of the mold, as well as the form of application of hard objects inside or under the mold. Excessive topical pressure with hard noncompressible tubing may lead, in extreme cases, to ischaemia and necrosis [Figure 4a-b].

Kairinos et al. have also challenged previous reports claiming increased blood flow by RNPT based on Doppler measurements. They suggest that the application of negative pressure to the wound may, initially, partially occlude the underlying compressible vessels with the compensating increase in blood velocity in the wound's capillaries, misinterpreted by laser Doppler readings as "increased perfusion". Kairinos et al. therefore suggested that laser Doppler is unsuitable for measurements of tissue perfusion under RNPT. ${ }^{|9|}$

This positive compression pressure inflicted on tissues by circumferential or topical RNPT dressings may be of considerable and, at times, of critical clinical significance in patients with predisposed peripheral vascular disease, with reduced ankle-brachial ratio (below 0.5 ) and low tissue perfusion pressure, as well as in treatment of traumatized tissue with compromised blood circulation.

Either ankle blood pressure measurements or Doppler monitoring, while recognizing their limitations, should precede and monitor RNPT in ischaemia predisposed treated areas. A lower work/pause time ratio in the intermittent or

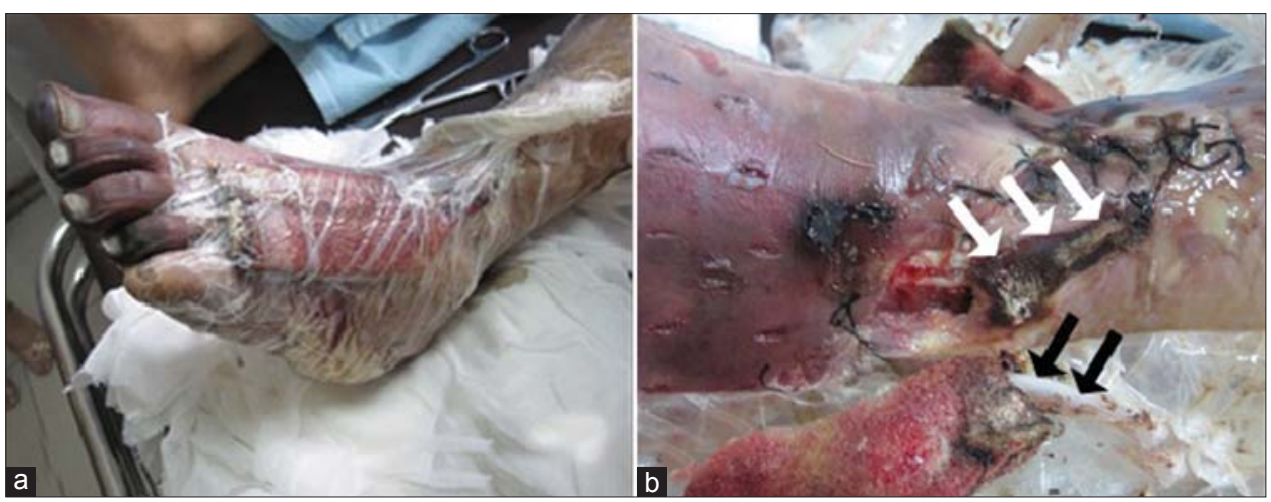

Figure 4: (a) Crush degloving injury to the foot following a road accident. Patient was treated with wall suction (300 mmHg) (b) Vacuum was connected to the sponge with a tube inserted at the bottom of the sponge inflicting excessive point topical pressure to the damaged tissue underneath, leading to necrosis (black arrows pointing at the tube and white arrows to the affected necrotic tissue) 
cyclic continuous RNPT operation modes should be applied to the wound to reduce ischaemia time and increase relative reperfusion time in order to reduce potential damage.

\section{Shearing forces}

Intermittent operation of RNPT was reported to improve wound healing and blood flow in animal studies, as well as in clinical applications, by stimulating angiogenesis and granulation tissue,$^{[5,10,11]}$ but an explanation for the mechanism involved has not been provided.

Chin et al. demonstrated, in a mouse model, the effect of a servo-controlled skin-stretching device inducing predetermined cyclical stretching forces on skin growth. ${ }^{[12]}$ It was demonstrated that the application of cyclical tensile force generated transient, alternating hypoxia and reperfusion that led to accelerated tissue growth. These mechano-transduction signaling pathways were found to upregulate principal growth factors, skin perfusion, angiogenesis and epidermal proliferation.

Intermittent operation of RNPT relay cyclical shearing forces and deformation to the underlying wound surface. Thus, one of the basic mechanisms that induce wound healing by intermittent negative pressure is associated with cyclical stretching forces on the underlying tissues generating mechano-transduction signaling that may enhance wound healing.

\section{Modification of wound atmospheric composition}

Lowering of atmospheric pressure decreases $\mathrm{pO}_{2}$ in accordance with Dalton's Law and may affect RNPT in the same manner. In order to confirm this hypothesis and to better understand the physiology of RNPT, an in vitro experimental model was designed to determine the spectrum levels of $\mathrm{pO}_{2}$ in the environment of wounds treated with RNPT and oxygen-enriched RNPT. ${ }^{[13]} \mathrm{pO}_{2}$ was measured at various oxygen flows and negative pressure ranges as indicated in clinical applications for wound treatment. $\mathrm{pO}_{2}$ values in the RNPT investigated system revealed a linear decrease of $\mathrm{pO}_{2}$ values, in direct correlation with the subatmospheric pressure that was applied to the simulated wounds. Simultaneous administration of oxygen to the system in the RO-NPT setup increased the $\mathrm{pO}_{2}$ measured under a clinical range of applied subatmospheric pressure, in direct correlation with oxygen inflow until reaching a plateau.

Anaerobes proliferate in an oxygen-depleted environment and can be a major setback to the healing process of various wounds, becoming a potentially life-threatening risk in severe complicated cases such as necrotizing fasciitis (NF). ${ }^{[14]}$ Numerous publications concerning the sensitivity of anaerobic bacteria to oxygen have suggested that the size of growth-inhibition zones is directly proportional to the logarithm of the partial pressure of oxygen used. ${ }^{[15,16]}$

$\mathrm{NF}$ is a life-threatening, rapidly progressive bacterial infection spreading through the deep fascial plane, with secondary necrosis of the fascia and subcutaneous tissues. $^{[17]}$ Once NF is suspected, empirically, broadspectrum antibiotics must be administered immediately. Early, aggressive, and frequent surgical debridement and exploration of necrotic tissue is essential, combined with intensive medical care and $\mathrm{HBO}$ as an adjunctive therapy. ${ }^{[18]} \mathrm{HBO}$ is considered standard treatment in cases where anaerobic infection of soft tissue is documented or suspected. ${ }^{[19]}$ In light of the above, those sporadic reports suggesting treatment of NF with $\mathrm{VAC}^{[20,21]}$ should be reassessed. Type $1 \mathrm{NF}$ is an example of an infection involving anaerobic bacteria in which RNPT should be contraindicated, where RO-NPT may be of imperative clinical significance, making it an alternative or, at times, preferable treatment to intermittent tissue oxygenation with HBO. The concept of topical oxygen therapy as an alternative to $\mathrm{HBO}$ has been acknowledged. Not only is it potentially less toxic, it may also be much more accessible and convenient, less expensive, and have few, if any, complications. ${ }^{[22]}$

The few case reports that relate the development of infection associated with RNPT, recommend the discontinuation of RNPT in anaerobic wound infections. ${ }^{[23 \mid}$ In order to minimize this complication, it was recommended to use VAC only on clean healthy wounds, thus limiting the scope of indications for RNPT. None of the reports, however, refer to the basic physical laws of gases to substantiate this recommendation. The optional advantages of simultaneously joining the two strategies of supplemental oxygen together with subatmospheric pressure for the combined prevention and treatment of anaerobic wound infections and the enhancement of wound healing seem to have significant merit. Laboratory measurements, jointly with positive wide clinical experience, point to the benefits of enriching the wound atmosphere with oxygen. Oxygen levels in the wound, treated with RO-NPT, can be monitored, calculated, and adjusted by altering oxygen inflow and/or subatmospheric pressure to attain the desired $\mathrm{pO}_{2}$ level. Careful handling 
of oxygen supplement to the wound is mandatory to avoid risk of flammability and combustion. RO-NPT may routinely be applied in NF cases, as prophylaxis in major trauma injuries, and in diabetic and PVD wounds.

\section{CLINICAL APPLICATIONS OF RNPT AND RO-NPT}

\section{Indications}

RNPT was originally applied to enhance healing of chronic wounds for treatment of diabetic foot and PVDaffected limbs. Indications have been widened to include neuropathic, postirradiation, and pressure sores. RNPT has been extensively applied in treatment of major trauma, in wide tissue losses, for treatment of open fractures; for various surgical applications, including dehiscence of surgical wounds, postoperative wound infections, and complications of failed sternal closures; in destructive deep burn injuries, deep and partial-thickness small to medium-sized burns; and for the enhancement of skin graft and flap survival.

There is a special indication for treatment of RNPT in crush injury and compartment syndrome. RO-NPT was designed for the prevention of anaerobic wound infections and as a supplemental mode of treatment of apparent anaerobic infections. Currently, the author is using RO-NPT for most major trauma, infected surgical wounds, and chronic wounds that are normally treated with negative pressure.

\section{Contraindications}

Special precautions and restrictions should be applied when using RNPT with the principal contraindications are summarized below. ${ }^{[24,25]}$ RNPT is contraindicated in conditions where acute uncontrolled bleeding may occur. A meticulous haemostasis should be established prior to the application of RNPT. The care provider should insure that no exposed blood vessels, nerves, or internal organs are in direct contact with the vacuum system. RNPT should be restricted in patients with open wounds who are treated with anticoagulants. Ulcerated malignant wounds are contraindicated for treatment by RNPT as vacuum treatment may accelerate tumour growth within the wound cavity. RNPT is contraindicated for treatment of unexplored fistulas. As mentioned above, RNPT should not be applied with apparent anaerobic infection, where RO-NPT should be used.

The care provider should not leave a wound covered for an extended time without effective negative pressure in order to avoid bacterial overgrowth and superinfection. Osteomyelitis was considered to be a contraindication for RNPT treatment with no apparent reasoning. The author has substantial experience treating these conditions with RNPT and RO-NPT with significant success.

RNPT should be restricted to small- and medium-sized burns or to a limited area of wide burns, as treatment of big area of deep or partial-thickness burns may lead to extensive extracellular fluid loss and electrolyte imbalance by the applied vacuum.

\section{Suction forces and acute bleeding}

Negative pressure has not infrequently been applied to a wound by direct connection to wall suction or to portable nondedicated suction machines, devices regulating vacuum levels of suction only [Figure 5].

By using wall suction or regular suction machines, the wound may be exposed to continuous negative pressures of 300 $\mathrm{mmHg}$ and over, with limited or no regulation of negative pressure and no hazards indicators, alluding to various important physiological consequences with potential catastrophic outcome of uncontrolled, undetected bleeding, into typically large volume canisters. In November 2009, the FDA issued a preliminary Public Health Notification of six deaths and 77 serious complications associated with negative pressure wound therapy systems over a period of 2 years prior to the notification. ${ }^{[26]}$ In its notification, healthcare practitioners were especially alerted to bleeding and infections associated with the use of negative pressure wound therapy (NPWT) systems, and were provided with recommendations to reduce the risk. Although rare, these complications can occur wherever NPWT systems are used, including acute and long-term healthcare facilities and at home. This catastrophic yet expected complication should



Figure 5: Types of suction used for NPT (a) Wall suction (b) Portable suction 
have been anticipated to occur with any negative pressure system that is applied to the wound. It would be logically anticipated that the greater the vacuum applied to the wound the greater the risk of bleeding. Bleeding should be anticipated when treating post-traumatic, postoperative, recently debrided wounds, in RNPT and RO-NPT systems applied to exposed organs or vessels and in patients with compromised coagulation systems (patients treated with anticoagulants or patients with congenital or acquired clotting disorders). Strict measures should be taken in order to avoid uncontrolled bleeding. Evaluation of risk/ benefit should precede RNPT and RO-NPT. Meticulous haemostasis should be confirmed with continuous monitoring of the patient's haemodynamic status and wound. The minimal effective negative pressure that would be clinically beneficial should be applied. RNPT devices should incorporate systems that indicate active bleeding, induce immediate cessation of suction and initiate instant audio-visual alarms that can alert both care provider and patient to possible active bleeding.

\section{CASE PRESENTATIONS OF CLINICAL USE OF RNPT AND RO-NPT}

\section{Case 1}

A33-year-old patient was admitted following a motorcycle accident, sustaining compound fractures of the tibia and fibula. The fractures were stabilized with external fixator and severe compartment syndrome required lateral and medial leg fasciotomy [Figures $6 \mathrm{a}, \mathrm{b}]$. The RNPT system was applied to the wound after meticulous haemostasis, to reduce oedema and contamination, simultaneously with TopClosure ${ }^{\circledR}$ skin stretching and secure system [Figures $6 \mathrm{~b}, \mathrm{c}$ ]. Skin was closed gradually, stretching the outer skin with TopClosure ${ }^{\circledR}$ combined with tension sutures that run on top of the TopClosure ${ }^{\circledR}$ plates to prevent direct pressure of the sutures on the skin, thus avoiding the common additional visible horizontal scars [Figure 6d]. The combined use of the RNPT system and TopClosure ${ }^{\circledR}$ to reduce infection and to gradually approximate wound edges eliminates both the need for skin grafting and for secondary aesthetic surgery [Figures 6e,f].

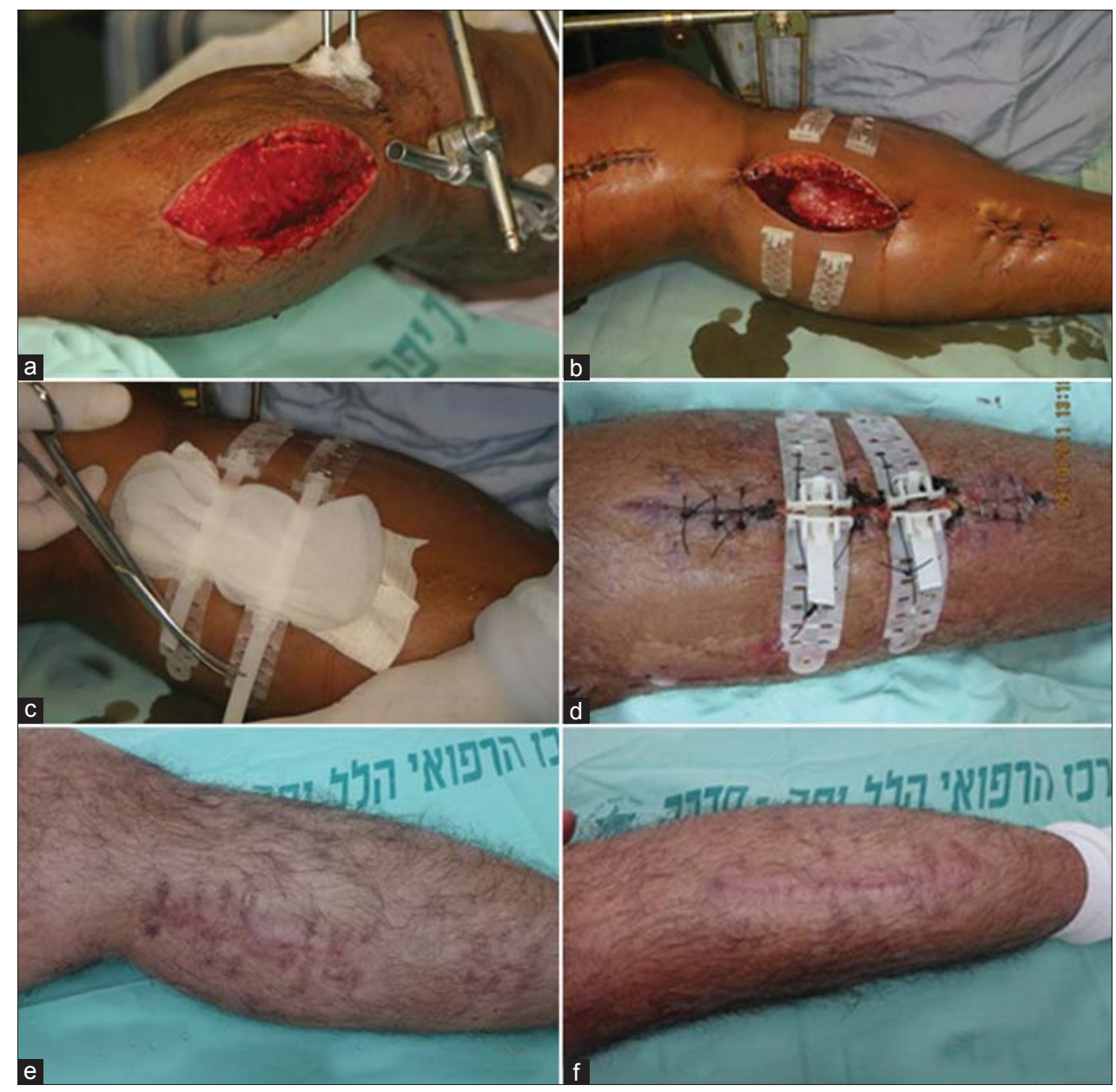

Figure 6: Case 1. Application of RNPT in compartment syndrome 
Typical closure of fasciotomy would be closing one side with gradual direct approximation of skin edges and the closure of the other side with skin graft. In this case, staged closure of both fasciotomy scars with TopClosure ${ }^{\circledR}$ enabled direct closure of both wounds without the use of skin graft at all.

\section{Case 2}

An 81-year-old woman was admitted due to a motor vehicle accident, suffering from extensive damage to her right upper limb and chest wall. Due to wide damage to the upper limb, disarticulation was performed, leaving a large cavity extending below the right breast, down to the right upper abdominal quadrant [Figure 7a]. Following recurrent sepsis episodes due to inadequate drainage of the infected wound, RNPT was applied. The sponge was inserted into the wound cavity, enabling

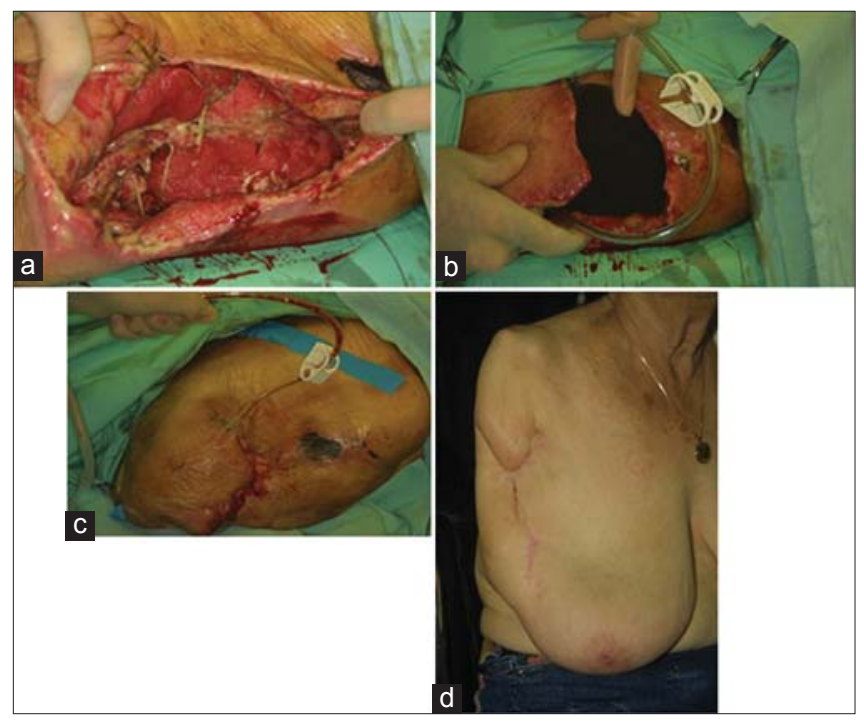

Figure 7: Case 2. Application of RNPT in heavily infected wounds ideal, effective drainage of the entire wound cavity surface [Figures $7 b-c]$. Change of wound dressing was performed regularly in the operating room. No further episodes of sepsis were observed following treatment with RNPT. Gradual extraction of the sponge from the wound allowed sequential obliteration of the wound cavity until complete wound edge approximation and healing [Figure $7 \mathrm{~d}$ ]. RO-NPT would have been a preferable choice of treatment today.

\section{Case 3}

A 36-year-old man sustained a crush thermal industrial injury (by a hot wheel) of his forearm [Figure 8a]. The patient underwent early wound debridement [Figure 8b] followed by RO-NPT as a prophylactic treatment for anaerobic infections [Figure 8c]. Early granulation tissue growth with no episodes of systemic or local infection were observed, enabling quick grafting and healing of the wound without hypertrophy or limitation in range of motion [Figures 8d-f].

\section{Case 4}

A 20-year-old man sustained $2^{\text {nd }}$ degree burn of his lower limbs following chemical burns due to misuse of fat removal cleaning material. The burn was treated by thorough irrigation with saline and early application of RNPT to both affected limbs [Figures 9a-b]. The course of healing was remarkably fast without signs of local or disseminated infection [Figures 9d-e]. RNPT may enable fast healing of burn wounds with improved scaring.

\section{Case 5}

A 71-year-old obese female with insulin-treated type 2 diabetes, presented with cellulitis and gangrene of her

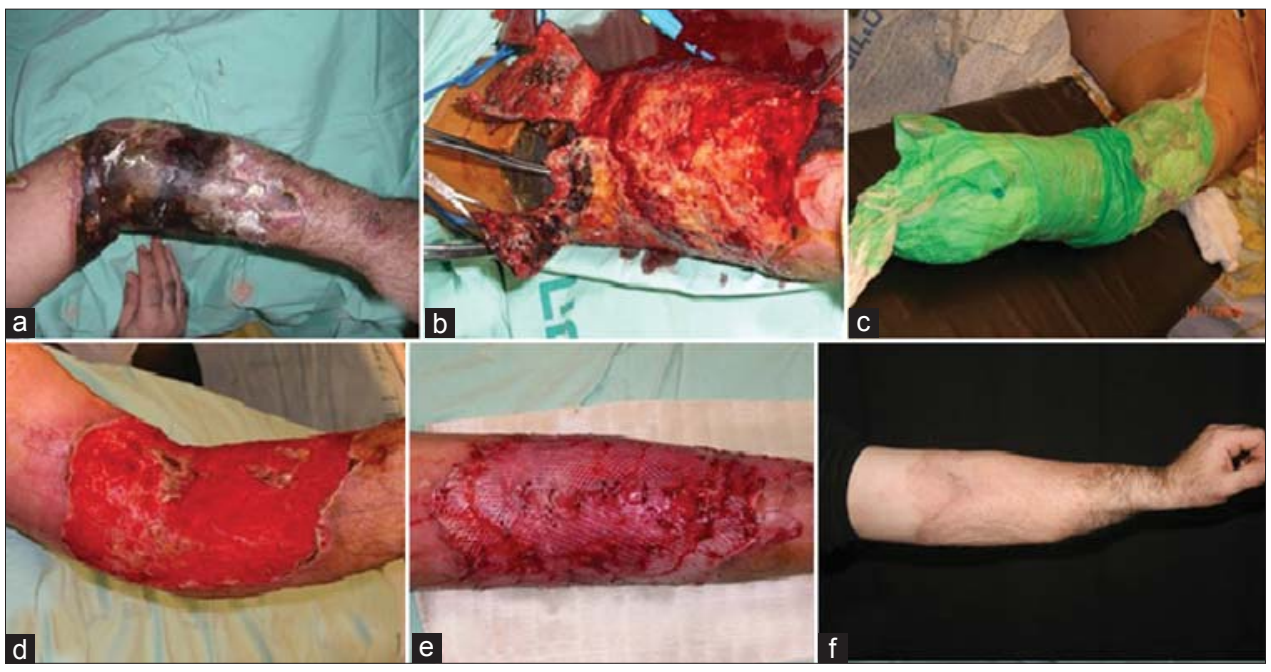

Figure 8: Case 3. RO-NPT for treatment of crush-burn wounds 
$2^{\text {nd }}$ toe. Following amputation of the toe [Figure 10a], vacuum treatment was commenced. The wound was closed gradually, assisted by incremental approximation of wound edges by TopClosure ${ }^{\circledR}$ under RNPT [Figures 10b,c]. RNPT should be regarded as a substantial tool for closure of diabetic ulcers restricting the application of low vacuum for avoiding topical ischaemia. Complete healing of the wound on both the dorsal and plantar aspects of the foot is shown [Figure 10d].

\section{Case 6}

RNPT treatment is an important tool for hard to heal wounds that are associated with some degree of pressure ulcers. It is of unique importance in dealing with elderly patients confined to bed with various pressure ulcers [Figures 11a,b]. In this group of patients, surgical procedures may pose lifethreatening risks that impose conservative treatment for most of these wounds. The application of TopClosure ${ }^{\circledR}$ and RNPT may enable bed-side, minimal surgical procedures to close this kind of wound, thus reducing surgical risks. In this patient, conservative, bed-side debridement of the wound was performed with combined treatment of RNPT and TopClosure ${ }^{\circledR}$ to clean, stretch and secure the skin [Figure 11c], followed by total excision of the wound under local anaesthesia with primary closure [Figure 11d]. This extends the indications of the technology for treatment of previously abandoned cases.

\section{Recommended RNPT operational modes, vacuum pressure level range, span, frequency of dressing changes}

Vacuum pressure in RNPT should be tailored to each specific wound and adjusted according to the patient's clinical condition. Greater negative pressure should be strictly avoided during the early stage of trauma treatment. Severe, life-threatening bleeding may result from the application of RNPT in the treatment of acute trauma, immediately after surgery, and especially in the early stages following debridement of wounds.

The past determination of $125 \mathrm{mmHg}$ as the ideal negative pressure and its recommendation for use should be regarded as obsolete and should be ignored. The higher range of negative pressures should be regarded as an ischaemia-inducing setting that may aggravate clinical ischaemic conditions such as in peripheral vascular disease, diabetic foot, and traumatised tissue. Excessive topical pressure may lead to compromised blood circulation and impairment of wound healing together with greater risk of bleeding. We have been applying lower negative pressure

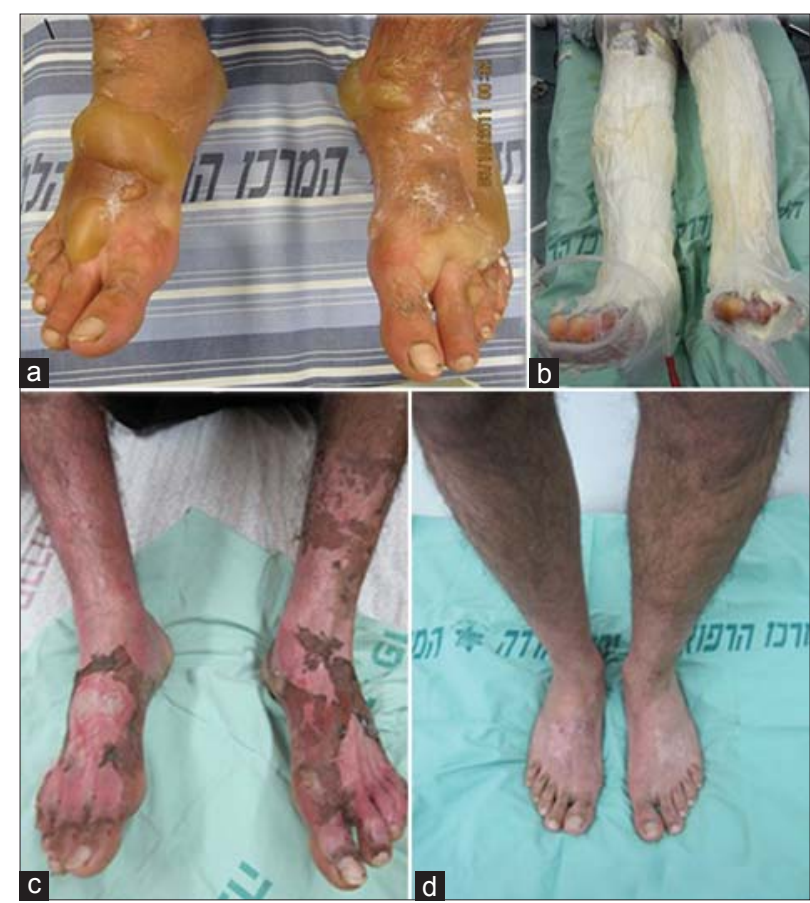

Figure 9: Case 4. RNPT in chemical burns



Figure 10: Case 5. Combined RNPT and TopClosure in management of diabetic foot

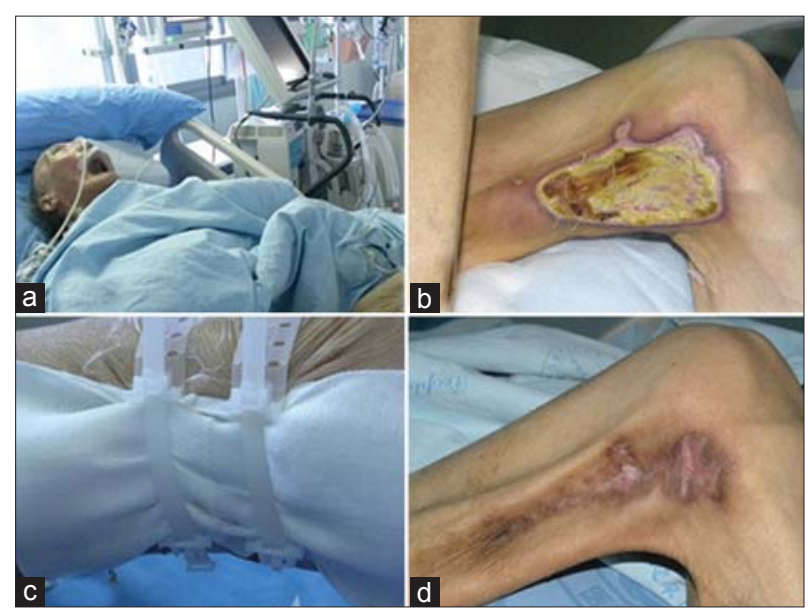

Figure 11: Case 6. Application of RNPT in pressure ulcers

Indian Journal of Plastic Surgery May-August 2012 Vol 45 Issue 2 
in the range of $60-80 \mathrm{mmHg}$ in the intermittent mode, for routine treatment of diabetic foot and PVD-induced limb ulcerations. Patients with an ankle-brachial ratio less than 0.5 should be treated with the lowest effective negative pressure and should be closely monitored for distal perfusion impairment, mainly when having circumferential RNPT dressing. When higher levels of negative pressure (deeper vacuum) are needed for the initial treatment of heavily contaminated wounds, tissue ischaemia can be managed and tolerated by short-term higher negative pressure values applied in an intermittent mode.

Our personal recommended vacuum pressures for various conditions and modes of application are presented in Table 1 . The practicing physician should recognize the risk for bleeding and infection and adjust settings for specific

Table 1: Proposed technical guidelines for clinical application of RNPT and RO-NPT*,**

\begin{tabular}{lcccc}
$\begin{array}{l}\text { Indication for } \\
\text { use - clinical } \\
\text { stages }\end{array}$ & $\begin{array}{c}\text { Working-pressure } \\
\text { range }(\mathrm{mmHg})\end{array}$ & $\begin{array}{c}\text { Operation modes: intermittent } \\
\text { (INTRM), continuous (CONT), } \\
\text { On/off min }\end{array}$ & $\begin{array}{c}\text { Rate of dressing } \\
\text { change (every- } \\
\text { days) }\end{array}$ & Remarks* $^{*}$ \\
\hline
\end{tabular}

Trauma

Acute phase $\quad 40-60$

Stable $70-120$

condition

Chronic wounds

Decubitus

$70-120$

ulcers

Peripheral vascular disease, diabetic foot

$\begin{array}{lc}\text { Acute } & 60-90 \text { INTRM } \\ & \text { (ONLY!) } \\ \text { Chronic } & 50-70 \text { INTRM }\end{array}$

(ONLY!)

$\begin{array}{ll}\text { Burns } & \\ \text { Acute } & 40-90 \\ \text { Nonacute } & 60-90\end{array}$

Burns

Nonacute

$60-90$

INTRM 2-3/1

INTRM 3-2/ 1

INTRM 2-3/1-2

1-2

2-4

INTRM 3-4/1-2

$2-5$

$50-80$
CONT for 2-3 days followed by
INTRM mode 6-4/1-2

INTRM 3-5/1-2 1-3

INTRM 3-5/1-2 2-4

\begin{abstract}
Stop active bleeding before starting RNPT treatment. Watch for active bleeding. Do not apply to blood vessels or internal organs. Apply lowest pressure possible in range. Increase pressure and frequency of dressing change for treatment of infected traumatic wounds. Apply nonadhesive antimicrobial dressing to the wound below the sponge. Intermittent mode is preferable.
\end{abstract}

Conservatively debride necrotic tissue prior to application of RNPT. Start treatment with greater vacuum in intermittent mode when wounds are heavily infected. Reduce pressure as the wound becomes cleaner. Consider combined systemic antibiotic treatment.

Correlate RNPT with measured anklebrachial pressure ratio. Use lowest pressure possible in range. Much greater negative pressure (100-120 $\mathrm{mmHg}$ ) may be applied for a few days for treating heavily infected wounds, applying a 2:1 ratio in intermittent mode and frequent dressing changes. Reduce pressure as soon as the wound becomes cleaner. Evaluate distal perfusion.

Limit application to small-medium-sized deep burns. Limit surface area to be treated according to the amount of fluid discharge from the wound. Stop treatment If excessive fluid is drained. Watch for acute bleeding. Determine electrolyte balance and evaluate need for systemic antibiotic treatment. Conservatively debride 3rd degree burns. Apply higher vacuum in range for heavily infected wounds.

$4-7$
Stop active bleeding before starting treatment. Use lowest pressure possible in range. Apply nonadhesive antimicrobial dressing to the wound below sponge.

\footnotetext{
*Safety measures regarding the use of RNPT should always be considered and implemented. Effective vacuum should be applied to the wound at all times in order to avoid super-infection. If vacuum is ineffective for over $30 \mathrm{~min}$, aeration of the wound by removal of occlusive dressing or another form of ventilation should be considered. Conservatively debride necrotic tissue prior to the application of RNPT. Alwaysconsider the necessity for RO-NPT for treatment or prevention of anaerobic infection. Evaluate the need for initiation and cessation of systemic antibiotic treatment in conjunction with RNPT, ${ }^{* *}$ The above technical guidelines should be regarded only as a general recommendation by the author. The treating physician should be knowledgeable with RNPT and RO-NPT and be qualified to amend and adjust treatment to match each individual clinical condition
} 
Topaz: RNPT and RO-NPT for improved wound management

vacuum pressure, work/pause ratio, and frequency of dressing changes according to the varying clinical conditions for each individual wound.

\section{SUMMARY}

RNPT should be regarded as a breakthrough technology in wound treatment and is the most important physical, nonpharmaceutical, platform technology developed and applied for wound healing in the last two decades. When properly applied, it is a simple, safe, and affordable tool that can be utilized in a wide range of acute and chronic conditions in medicine, surgery, orthopedics, plastic surgery, vascular surgery, etc., with reduced need for antibiotics and complicated surgical procedures, and lower cost. This technology has been shown to be effective and safe, saving limbs and lives on a global scale. RO-NPT is an innovative technology, whereby supplemental oxygen is simultaneously administered with RNPT for their synergistic effect on treatment and prophylaxis of anaerobic wound infection and promotion of wound healing. Understanding the basic science of these technologies through their fundamental clinical mechanisms is at the core of their improved application.

\section{REFERENCES}

1. Argenta LC, Morykwas MJ, Marks MW, DeFranzo AJ, Molnar JA, David LR. Vacuum assisted closure: State of clinic art. Plast Reconstr Surg 2006;117:127S-42S.

2. Topaz M. Chapter 35: Role of regulated negative pressure therapy in wound healing. In: Sarabahi S, Tiwari VK, editors. Principal and practice of wound care. Jaypee Bros Pubs, New Delhi, India; 2012;401-31.

3. Moues CM, Vos MC, Van den Bemd G, Stijnen T, Hovius SE. Bacterial load in relation to vacuum-assisted closure wound therapy: A prospective randomized trial. Wound Repair Regen 2004;12:11-7.

4. Wagner S, Coerper S, Fricke J, Hunt TK, Hussain Z, Elmlinger MW, et al. Comparison of inflammatory and systemic sources of growth factors in acute and chronic human wounds. Wound Repair Regen 2003; $11: 253-60$

5. Morykwas MJ, Argenta LC, Shelton-Brown El, McGuirt W. Vacuumassisted closure: A new method for wound control and treatment. Animal studies and basic foundation. Ann Plast Surg 1997;38: 553-62.

6. Fabian TS, Kaufman HJ, Lett ED, Thomas JB, Rawl DK, Lewis PL, et al. The evaluation of subatmospheric pressure and hyperbaric oxygen in ischemic full-thickness wound healing. Am Surg 2000;66:1136-43.

7. Morykwas MJ, Faler BJ, Pearce DJ, Argenta LC. Effects of varying levels of subatmospheric pressure on the rate of granulation tissue formation in experimental wounds in swine. Ann Plast Surg 2001;47:547-51.

8. Chen SZ, Li J, Li XY, Xu LS. Effects of vacuum-assisted closure on wound microcirculation: An experimental study. Asian J Surg 2005;28:211-7.

9. Kairinos N, Voogd AM, Botha PH, Kotze T, Kahn D, Hudson DA, et al. Negative-pressure wound therapy II: Negative-pressure wound therapy and increased perfusion. Just an illusion? Plast Reconstr Surg 2009;123:601-12.

10. Sjögren J, Gustafsson R, Koul B, Ingemansson R. Selective mediastinal tamponade to control coagulopathic bleeding. Ann Thorac Surg 2003;75:1311-3.

11. llizarov GA. The tension-stress effect on the genesis and growth of tissues. Part I. The influence of stability of fixation and soft-tissue preservation. Clin Orthop Relat Res 1989;238:249-81.

12. Chin MS, Ogawa R, Lancerotto L, Pietramaggiori G, Schomacker $\mathrm{KT}$, Mathers JC, et al. In vivo acceleration of skin growth using a servo-controlled stretching device. Tissue Eng Part C Methods 2010;16:397-405.

13. Topaz M, Bisker O, Litmanovich M, Keren G. Application of regulated oxygen-enriched negative pressure-assisted wound therapy in combating anaerobic infections. Eur J Plast Surg Published online 2010 Oct 21.

14. In: Finegold SM, George WL, editors. Anaerobic Infections in Humans. San Diego, CA: Academic Press; 1989.

15. Fredette $V$, Plante $C$, Roy $A$. Numerical data concerning the sensitivity of anaerobic bacteria to oxygen. J Bacteriol 1967;93:2012-7.

16. Loesche WJ. Oxygen sensitivity of various anaerobic bacteria. Appl Microbiol 1969;18:723-7.

17. Zahar JR, Goveia J, Lesprit P, Brun-Buisson C. Severe soft tissue infections of the extremities in patients admitted to an intensive care unit. Clin Microbiol Infect 2005;11:79-82.

18. Kaide CG, Khandelwal S. Hyperbaric oxygen: Applications in infectious disease. Emerg Med Clin North Am 2008;26:571-95.

19. Gottrup F. Oxygen in wound healing and infection. World J Surg 2004;28:312-5.

20. de Geus HR, van der Klooster JM. Vacuum-assisted closure in the treatment of large skin defects due to necrotizing fasciitis. Intensive Care Med 2005;31:601.

21. Oczenski W, Waldenberger F, Nehrer G, Kneifel W, Swoboda H, Schwarz S. Vacuum-assisted closure for the treatment of cervical and mediastinal necrotizing fasciitis. J Cardiothorac Vasc Anesth 2004;18:336-8.

22. Rodriguez PG, Felix FN, Woodley DT, Shim EK. The role of oxygen in wound healing: A review of the literature. Dermatol Surg 2008;34:1159-69.

23. Gwan-Nulla DN, Casal RS. Toxic shock syndrome associated with the use of the vacuum-assisted closure device. Ann Plast Surg 2001;47:552-4.

24. V.A.C. Therapy indications and contraindications. Available from: http://www.kci1.com/KCl1/indications and contraindications. [Last accessed August 6, 2012.]

25. FDA Preliminary Public Health Notification*: Serious complications associated with negative pressure wound therapy systems. Available from: http://www.fda.gov/Medical Devices/Safety/Alerts and Notices/Public Health Notifications/ucm190658.htm. [Last accessed August 6, 2012.]

26. U.S. Food and Drug Administration. Preliminary Public Health Notification: Serious complications associated with negative pressure wound therapy systems (FDA web site). Date: November 13, 2009. Available from: http://www.fda.gov/Medical Devices/ Safety/Alerts and Notices/Public Health Notifications/ucm190658. htm. [Last accessed August 6, 2012.].

How to cite this article: Topaz M. Improved wound management by regulated negative pressure-assisted wound therapy and regulated, oxygen- enriched negative pressure-assisted wound therapy through basic science research and clinical assessment. Indian J Plast Surg 2012;45:291-301.

Source of Support: Nil, Conflict of Interest: M. Topaz is among the developers of the TopClosure ${ }^{\circledR} 3 S$ and the Healer $\alpha^{\circledR}$ RNPT systems. .

Indian Journal of Plastic Surgery May-August 2012 Vol 45 Issue 2 